\title{
Viral hepatitis in Germany: poor vaccination coverage and little knowledge about transmission in target groups Karl Schenkel*1, Doris Radun ${ }^{1}$, Viviane Bremer ${ }^{1}$, Nikolaus Bocter ${ }^{2}$ and Osamah Hamouda ${ }^{1}$
}

\author{
Address: ${ }^{1}$ Department for Infectious Disease Epidemiology, Robert Koch Institute, Berlin, Germany and ${ }^{2}$ Academy for Public Health Services, \\ North Rhine Westphalia, Germany \\ Email: Karl Schenkel* - schenkelk@rki.de; Doris Radun - radund@rki.de; Viviane Bremer - bremerv@rki.de; \\ Nikolaus Bocter - bocter@akademie-oegw.de; Osamah Hamouda - hamoudao@rki.de \\ * Corresponding author
}

Published: 23 April 2008

BMC Public Health 2008, 8:132 doi:10.1 186/147/-2458-8-132

This article is available from: http://www.biomedcentral.com/I47I-2458/8/I32

(c) 2008 Schenkel et al; licensee BioMed Central Ltd.

This is an Open Access article distributed under the terms of the Creative Commons Attribution License (http://creativecommons.org/licenses/by/2.0), which permits unrestricted use, distribution, and reproduction in any medium, provided the original work is properly cited.

\begin{abstract}
Background: In Germany, vaccination against hepatitis B is recommended for infants, children and adolescents since 1995 and for specific target groups since 1982. Little is known about knowledge about viral hepatitis and attitudes toward hepatitis B vaccination-factors likely to influence vaccine uptake.
\end{abstract}

Methods: In order to estimate vaccination coverage in adult target groups and in the overall adult population and to assess knowledge and attitudes, we conducted a nationwide cross-sectional telephone survey among 412 persons in November 2004. We defined participants as being vaccinated if they reported at least one previous vaccination against hepatitis $B$.

Results: Vaccination coverage (vc) standardised for age, sex and residence was $29.6 \%$ in the general population and $58.2 \%$ in target groups for hepatitis B vaccination. Particular gaps in vaccine coverage were detected among health care workers (vc: 69.5\%) and chronically ill persons (vc: $22.0 \%$ ). Knowledge on risk factors and transmission was far below expectations, whereas the acceptance of vaccination in the majority of the population (79.0\%) was good.

Conclusion: We conclude that educational measures could lead to a higher vaccination uptake in adult target groups.

\section{Background}

Worldwide, 300 to 420 million people are chronically infected with Hepatitis B (HB), affecting $5-7 \%$ of the worlds population $[1,2]$. About 2 billion people (nearly a third of the world's population) had previous contact with the hepatitis B virus, corresponding to past or current infection with hepatitis B.
Globally, more than 500.000 persons per year die from the consequences of chronic HB infection. Main modes of transmission are contacts with infected blood or other body fluids, for example during sexual intercourse. Vertical transmission also occurs, unless the newborn is vaccinated.

Compared to the overall, worldwide seroprevalence of markers for $\mathrm{HB}$, in Germany seroprevalence is low: $5-8 \%$ 
of the general population had a past or current HB infection and $0.4-0.7 \%$ are carriers of HBs-antigen, indicating acute or chronic infection, accounting for an estimated number of 300.000 to 650.000 potentially infectious people [3-6]. In 2004, 1260 cases of acute hepatitis B were notified, corresponding to an annual incidence of 1.5 cases/100,000 inhabitants per year, with the highest age group specific incidence in young adults [6]. At least 30\% of notified hepatitis B cases in 2003/2004 occurred in unvaccinated people belonging to target groups [7].

In 1982, the Standing Committee on Vaccination Recommendations in Germany (STIKO) recommended HB vaccination for people belonging to target groups, e.g. health care workers or other persons with potential occupational exposure to HB virus, travellers to high-prevalence-countries, people with chronic renal or liver disease, intravenous drug users or men who have sex with men [8]. In 1995, the STIKO recommendation was expended for all infants, children and adolescents to be vaccinated against HB [8]. Vaccination coverage among children is determined at school entry.

In 2004, 84\% of the 6 year-olds having their vaccination documents available at school entry were fully vaccinated against HB [9].

Populations in Germany at particular risk for hepatitis B virus infection have been estimated to amount to 1.85 million healthcare workers, about 1 million men who have sex with men, 100000 injecting drug users, and more than 50000 haemodialysis patients [3]. However, data on HB-vaccine coverage among these target groups is sparse.

It is also unknown whether insufficient awareness and knowledge of $\mathrm{HB}$ transmission and possible protective measures may form a major obstacle in terms of following to vaccination recommendations in target groups [10]. For a majority of the target groups such as for medical staff the responsibility to immunise lies with employer's health care providers. Nevertheless, there is no obligatory duty to get immunised for medical staff so far. Thus, it can be concluded that individual knowledge and perceived risk for HB infection play a key role for vaccination uptake in these groups.

In order to assess the current vaccination coverage in the general population and to learn more about knowledge on viral hepatitis and attitudes towards $\mathrm{HB}$ vaccination in the adult population in Germany, particularly in target groups for $\mathrm{HB}$ infection we initiated a cross-sectional nationwide telephone survey.

\section{Methods}

We conducted a nationwide telephone survey on November 8th and 29th 2004, involving participants of a course on interventional infectious disease epidemiology as interviewers. We aimed for a sample size of 350 interviewees, according to calculations based on an estimated HB vaccine coverage among target groups of $30 \%$ at a precision of $+/-1.7 \%$.

We used an anonymised, standardised questionnaire consisting of mainly closed questions regarding vaccination coverage and knowledge about viral hepatitis symptoms, transmission modes and existing prevention measures, as well as the general attitude towards vaccination. Interviewers were briefed and trained in advance of the survey.

Random telephone numbers following the method by Gabler and Häder were used to contact interviewees [11]. This method for achieving equivalence of samples in cross-national surveys allows a maximum inclusion opportunity for all fixed telephone subscribers in Germany.

We estimated the prevalence of having an indication for $\mathrm{HB}$ vaccination amongst the population of $10 \%$. In order to reach a precision of at least $3 \%$ at an alpha level of $95 \%$ and a statistical power of $80 \%$ we calculated a minimum sample size of 384 completed interviews.

We oversampled participants from East Germany (the former German Democratic Republic), where only $17 \%$ of the adult German population reside, in order to achieve sufficient statistical power and to reduce random error.

All results relating to the entire country are given as standardised data (age group, sex and geographic origin), whereas data referring to particular subgroups are displayed as raw numbers.

We standardized the results according to age groups (1829 years, 30-39 years, 40-49 years, 50-59 years, ? 60 years), sex and geographical origin (West or East Germany). We assigned Berlin to West Germany. Standardisation was used for the overall population in Germany but also for specific target groups for HB vaccination (such as travellers, medical professionals etc.).

The National Bureau for Statistics in Germany provided us with information on population density and structure in West and East Germany as of 2003.

Every person of at least 18 years of age contacted and willing to participate who understood and spoke German satisfactorily was considered eligible. In order to avoid selection bias, we inquired for the one member of the 
household fulfilling criteria of eligibility with the most recent birth date and asked him or her to participate. If only one person was present, we asked this person to participate. Informed oral consent about the selection process and data protection issues was obtained before every interview.

We defined a participant as being "vaccinated" if he or she reported at least one (lifetime) previous vaccination with a HB vaccine. Persons who did not know whether they had been vaccinated previously were considered as "not vaccinated".

We defined three main target groups for $\mathrm{HB}$ vaccination in accordance with current STIKO recommendations:

Persons with an occupational risk of HB infection (e.g. health care workers, social workers)

Persons with a travel history to a high-prevalence country for $\mathrm{HB}$

Chronically ill, i.e. persons with an elevated risk for HB infection or enhanced risk of morbidity, e.g. those with chronic renal or liver disease

In addition to questions concerning the vaccination status of interviewees, we gained information about their knowledge concerning causative agents, modes of transmission and measures of protection against viral hepatitis and attitudes towards $\mathrm{HB}$ vaccination.

We processed and analysed data in EPI-Info 2002, version 4.02 April 2004 and SPSS Version 12.0. Besides descriptive analyses, we conducted group comparisons, bivariate analysis and a multivariate logistic regression (backward elimination) in order to detect variables independently associated with vaccination or knowledge about $\mathrm{HB}$, respectively.

In a first model, our outcome variable was "being vaccinated against $\mathrm{HB}$ ", and we included those variables associated with vaccination in the bivariate analysis with either an alpha level of ? 0.05 or a plausible association with the vaccination status.

A second model was designed in order to identify variables associated independently with "good knowledge" about viral hepatitis. For these purposes, we created a combined variable, indicating good knowledge as:

Knowing at least two of the three most important potential transmissions routes of $\mathrm{HB}$ virus (sexual transmission, blood transfusion, needle sharing) and

Knowing at least two correct answers on the three most important protection measures (vaccination, condom use, hygiene measures such as gloves and hand disinfection for caretakers of infected persons)

and

Recognizing at least three out of five hepatitis forms (Hepatitis A-E)

We included variables significantly associated with "good knowledge" in the bivariate analysis at a p level of ? 0.05 or a plausible association with the outcome variable.

Consent on data protection had been obtained in advance from the data security officer of the Robert Koch Institute. Ethical approval was not considered as participation was voluntary; we used anonymous data only and did not apply any invasive procedures (such as biological sample taking) on participants.

\section{Results \\ Study population}

Of 4864 computer generated random numbers dialled, (which, according to the Gabler/Häder method applied included an unknown proportion of non-existing numbers) we connected to 1431 (29.4\%) eligible persons. Of those, 412 persons $(28.8 \%)$ participated in the survey, of whom 241 participants (58\%) resided in West-Germany and $171(42 \%)$ in East-Germany.

With a sample population of 412 interviewees who completed interviews we reached a precision of $+/-2.9 \%$ at an alpha level of $95 \%$ and a statistical power of $80 \%$.

One hundred forty three (59.3\%) of West German participants and 106 (62\%) of East German participants were female. Median age was 44 years in West Germany (range 18-99 years) and 46 years in East Germany (range 18-74 years).

Overall, 93/412 participants (22.9\%) belonged to at least one target group for $\mathrm{HB}$ vaccination, among them 59 from the West (24.5\% of all West German participants) and 34 from the East (19.9\% of all East German participants). We failed to obtain information on whether interviewees belonged to a target group in $6 / 412$ cases (1.5\%).

Altogether, $49.1 \%$ of the participants belonging a target group reported having had a risk related to travel, $45.1 \%$ reported occupational risks and $14.9 \%$ stated they had a risk related to chronic disease (multiple answers were allowed). 


\section{Vaccination coverage}

Information on vaccination status was available in 406 persons (98.5\% of participants): $26.3 \%$ (95\% CI $19.9-$ 33.6) of East-German participants and 31.1\% (95\% CI 25.2-37.4) of West German participants were vaccinated according to our definition.

After standardisation for sex, age group and residency (West/East Germany), this corresponded to an overall HB vaccination coverage of $29.6 \%$ (95\% CI 23.3-34.4) in Germany. A total of $55.7 \%$ of those vaccinated were female (95\% CI 46.5-64.7).

A total of $25.0 \%$ of participants belonged to at least one of three target groups for HB vaccination (95\% CI 20.729.3 ). Of those, $58.2 \%$ (95\% CI 47.9-67.7) were vaccinated according to our definition (table 1 ).

Median age for members of the target groups "travel risk", "occupational risk" or "chronic disease" was 37, 31, and 46 years respectively.

A higher vaccination coverage among all vaccinated was seen in persons with higher education levels (completed high school) in comparison to those with lower education levels (36.4\% versus $25.7 \%$, p < 0.05).

Of vaccinated participants, $41.2 \%$ reported having received at least three doses, $4.6 \%$ reported having had 2 doses and 3.5\% reported having had one dose of $\mathrm{HB}$ vaccine previously, whereas $49.1 \%$ did not recall the exact number of doses they had received (2 cases missing).

\section{Attitudes towards vaccination}

$151 / 171$ (88.3\%) participants from East Germany and $181 / 235$ (77.0\%) participants from West Germany had a positive attitude towards vaccinations in general. This corresponds to a standardised percentage of $79.0 \%$ (95\% CI 74.7-82.7) for the entire country.

Standardised results for Germany indicate that $76.7 \%$ of the participants (95\% CI 71.9-81.1) were generally aware of the availability of a vaccine against HB. Nearly two thirds $(66.2 \%)$ believed in a good protective efficacy of the vaccine (95\% CI 60.1-71.8) (figure 1).

\section{Knowledge about viral hepatitis, transmission modes and protective measures}

HB was known best, but only $57.7 \%$ actively mentioned it (table 2) when asked about different forms of hepatitis.

"Hepatitis B" could be named actively by $64 \%$ of participants with a secondary level school degree and by $93 \%$ of participants with A-level degrees $(\mathrm{p}<0.05)$.

Nearly $60 \%$ of the sample population identified $\mathrm{HB}$ as being caused by a virus (figure 2). No significant difference in knowledge on the pathogen causing $\mathrm{HB}$ was found between target groups versus those without explicit indication for $\mathrm{HB}$ vaccination.

While the majority of interviewees realized that jaundice is among the possible symptoms of $\mathrm{HB}$ infection, less than half of them were aware that $\mathrm{HB}$ can cause liver cancer (table 3).

Regarding knowledge about possible clinical outcomes of $\mathrm{HB}$, there was no relevant difference between all target groups for $\mathrm{HB}$ vaccination versus other population groups. However, the subgroup of those occupationally at risk compared to other population groups was more aware of possible sequelae of $\mathrm{HB}$ such as liver cancer, liver cirrhosis or liver failure (table 3 ).

Unprotected sexual intercourse was recognised as a risk factor more frequently in target groups for $\mathrm{HB}$ vaccination than in other participants $(75.6 \%$ vs. $50.8 \%$, p < 0.05$)$, whereas needle sharing and blood transfusion were identified as risk factors by all groups of participants.

Figure 3 gives an overview of knowledge about transmission modes for HB infection.

In the overall sample, $73.9 \%$ (95\% CI 68.9-78.4) recognised condoms as a protective measure to prevent $\mathrm{HB}$. Condoms were less well recognised as protective than $\mathrm{HB}$ vaccination and hygiene measures. Figure 4 summarises answers concerning knowledge about protective measures to avoid HB.

Table I: HB vaccination status according to target group, Germany. Standardised by age, sex and residence (East/West Germany), November $2004(n=412)$

\begin{tabular}{lcc}
\hline Target group & Vaccination coverage (\%) & 95\% Confidence Interval \\
\hline Occupational risks & 69.5 & $54.3-82.3$ \\
Travel to highly endemic countries for HB & 52.7 & $37.9-66.7$ \\
Chronic renal or liver disease & 22.0 & $4.4-48.4$ \\
At least one target group & 58.2 & $47.9-67.7$ \\
\hline
\end{tabular}




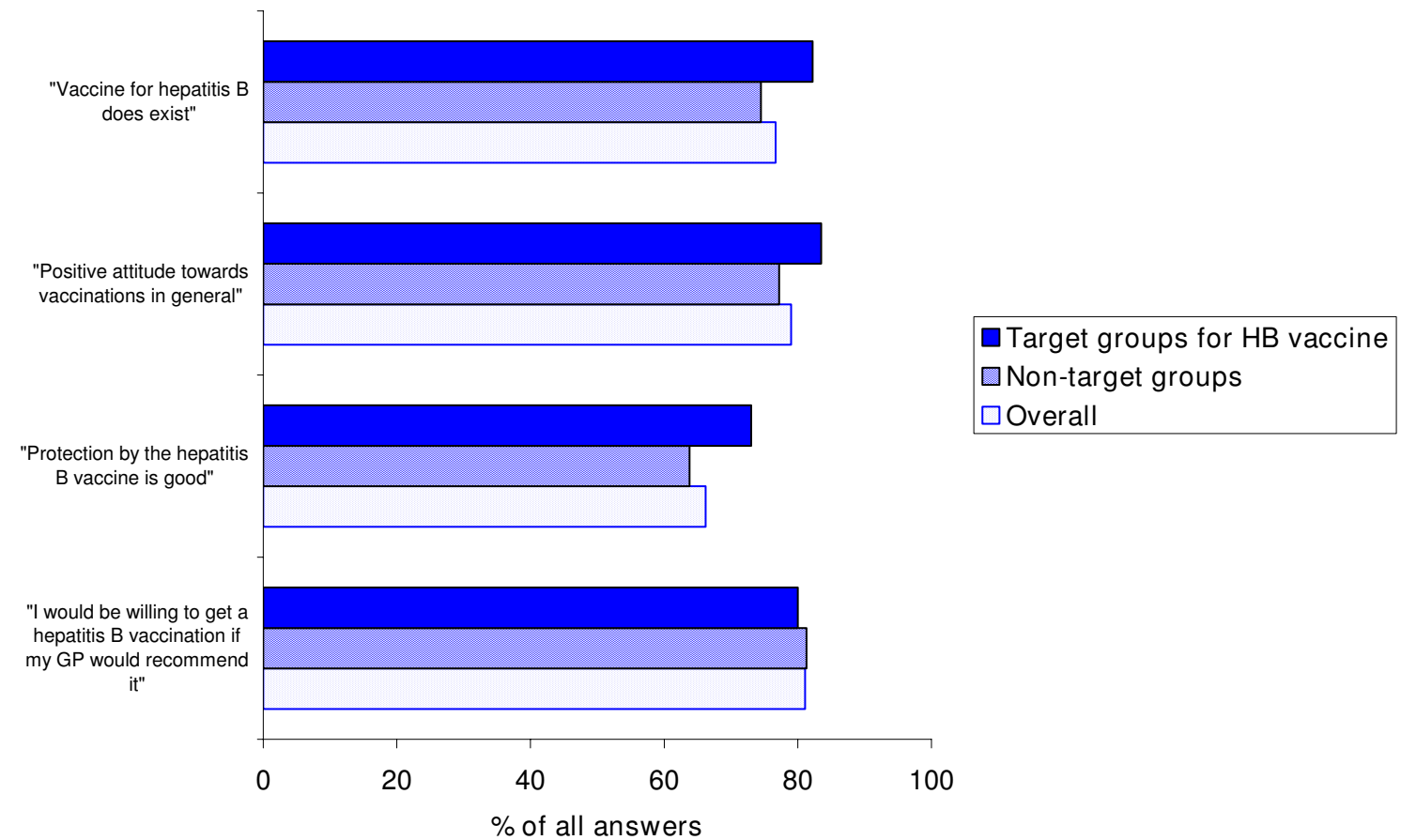

Figure I

Attitudes and opinions towards the HB vaccine, and vaccines in general. (All participants and target groups for $H B$ vaccination. Standardised results (sex, age group and regional distribution) for overall Germany, November $2004(n=4 I 2)$

Female and male study participants did not differ in their judgement of protective efficacy of condoms (51.8 vs. $48.2 \% \mathrm{p}>0.05)$.

\section{Associations with vaccination status}

Associations with vaccination status retrieved from bivariate analysis are shown in figure 5 . The associated factors identified hereby were later included into a multivariate analysis in order to detect factors independently associated with vaccination status.

Occupational risk for HB infection (OR 5.6, 95\% KI 2.015.8) and travel history (OR 5.4, 95\% KI 2.0-14.7) were independently associated with positive vaccination status. Age was inversely associated with vaccination status - the older participants were, the less likely they were to be vac- cinated (OR for incremental age group: 0.8 (95\% CI 0.6$0.9))$.

\section{Associations with "good knowledge"}

Occupational risks for HB infection (OR 11.5 (95\% CI 2.5-52.8)) and positive attitude towards vaccinations in general (OR 5.4 (95\% CI 1.2-19.9)) were independently associated with "good knowledge" (tables 4 and 5). Female gender was negatively associated with "good knowledge" (OR 0.5 (95\% CI 0.3-0.9)), but there was an interaction between gender and educational level (OR for interaction term "school leaving certificate (high school certificate/other)" * "sex (female/male)" 2.4 (95\% CI 1.24.6)). Furthermore, the older participants were, the less likely they were to have "good knowledge" (table 5).

Table 2: Active and passive nominations/recognition of viral hepatitis forms. Standardised results (sex, age group and regional distribution) Germany, November $2004(n=412)$

Hepatitis A (\%) Hepatitis B (\%) Hepatitis C (\%) others (\%)

\begin{tabular}{|c|c|c|c|c|}
\hline $\begin{array}{l}\text { „Have you ever heard about one of the following hepatitis forms? } \\
\text { (passive recognition) }\end{array}$ & 79.3 & 83.4 & 62.5 & 16.9 \\
\hline Which kinds of viral hepatitis do you know? (active recognition) & 54.3 & 57.7 & 40.5 & II.2 \\
\hline
\end{tabular}




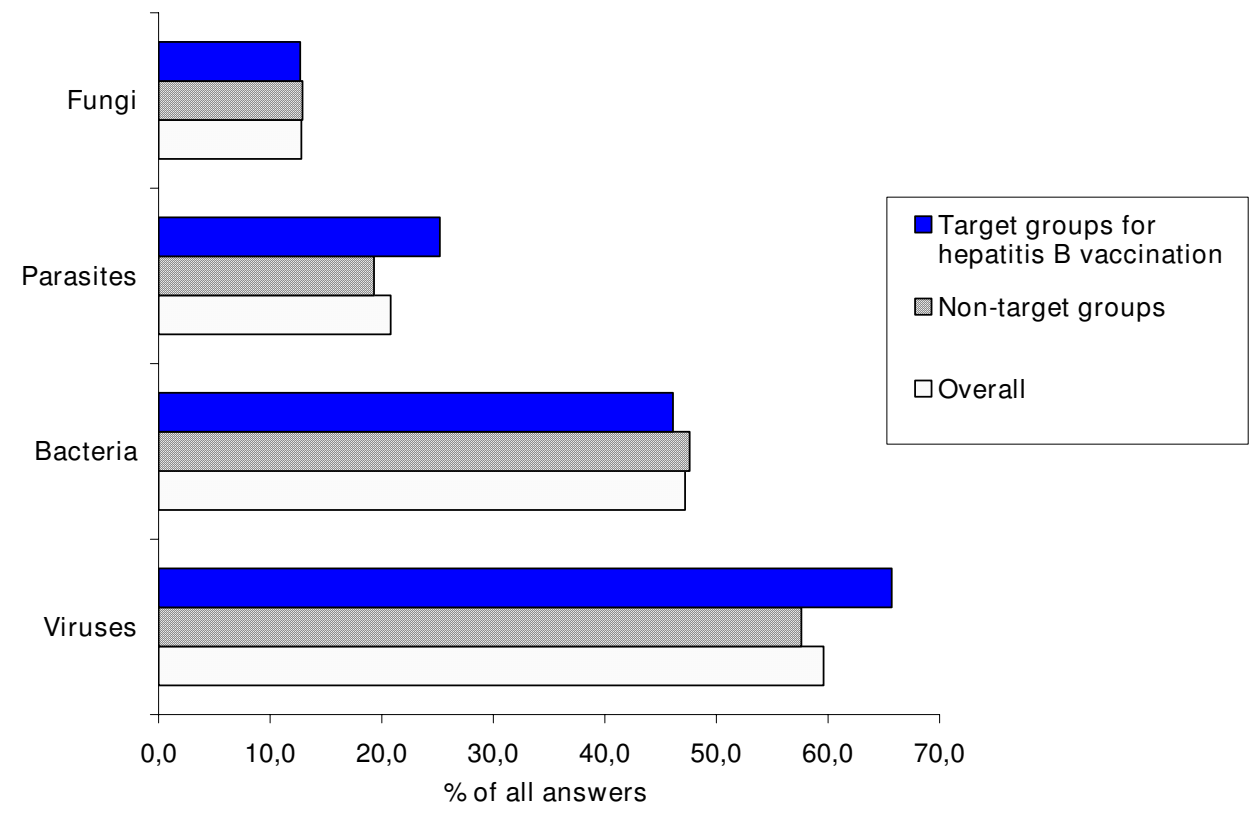

Figure 2

Identification of etiologic agents for HB in sample population. Standardised results (sex, age group and regional distribution), Germany, November 2004 ( $n=4 \mid 2)$

\section{Sources of information on vaccination issues}

The majority of interviewees (85\%) reported to have received information on vaccinations from their General Practitioners (GPs) who were identified as the most important information source for vaccine issues throughout all age groups. Other information sources like specialised doctors, alternative healers, pharmacies, radio, TV and internet were less frequently used, depending on age group (figure 6).

\section{Discussion}

We conclude that with a reached precision of $2,9 \%$ at an alpha level of $95 \%$ we covered a representative sample of the major target groups for the $\mathrm{HB}$ vaccination within the German population. Our study may have been biased by the fact that migrants with poor knowledge of German were underrepresented in the sample population. According to the Institute of Economic research about one third of migrants in Germany claim not to be capable of adequate German language skills [12]. This clearly represents a weakness of our study and means a tribute to the needs of a large-scale telephone interview.

We were able to gain insights into $\mathrm{HB}$ vaccine coverage among target groups and the general population, as well as on knowledge and attitudes regarding hepatitis $\mathrm{B}$. We observed a vaccination coverage of nearly $30 \%$ in the overall population and of around 60\% in target groups with a high variability among the three target groups.

Despite a comparatively low response, we believe that no major bias distorted our findings as the sampling methods allowed for recruiting a best possible sample in terms of representativeness.

Table 3: Known possible clinical symptoms or sequelae of HB infection. Standardised results (for sex, age group and regional distribution). Germany, November $2004(n=4$ I 2)

\begin{tabular}{lcccc}
\hline $\begin{array}{l}\text { Knowledge on clinical outcome of HB } \\
\text { infection }\end{array}$ & \% of all answers & $\mathbf{9 5 \%} \mathbf{C l}$ & $\begin{array}{c}\text { Answers from the target group of occupation- } \\
\text { ally exposed }\end{array}$ & $\begin{array}{c}\mathbf{9 5 \%} \mathbf{C l} \\
\text { Jaundice }\end{array}$ \\
Liver failure & 83.6 & $79.1-87.2$ & 87.1 & $73.1-95.8$ \\
Liver cirrhosis & 67.4 & $62.2-72.4$ & 91.8 & $79.5-98.4$ \\
Liver cancer & 60.8 & $55.4-66.0$ & 85.6 & $70.4-94.3$ \\
\hline
\end{tabular}




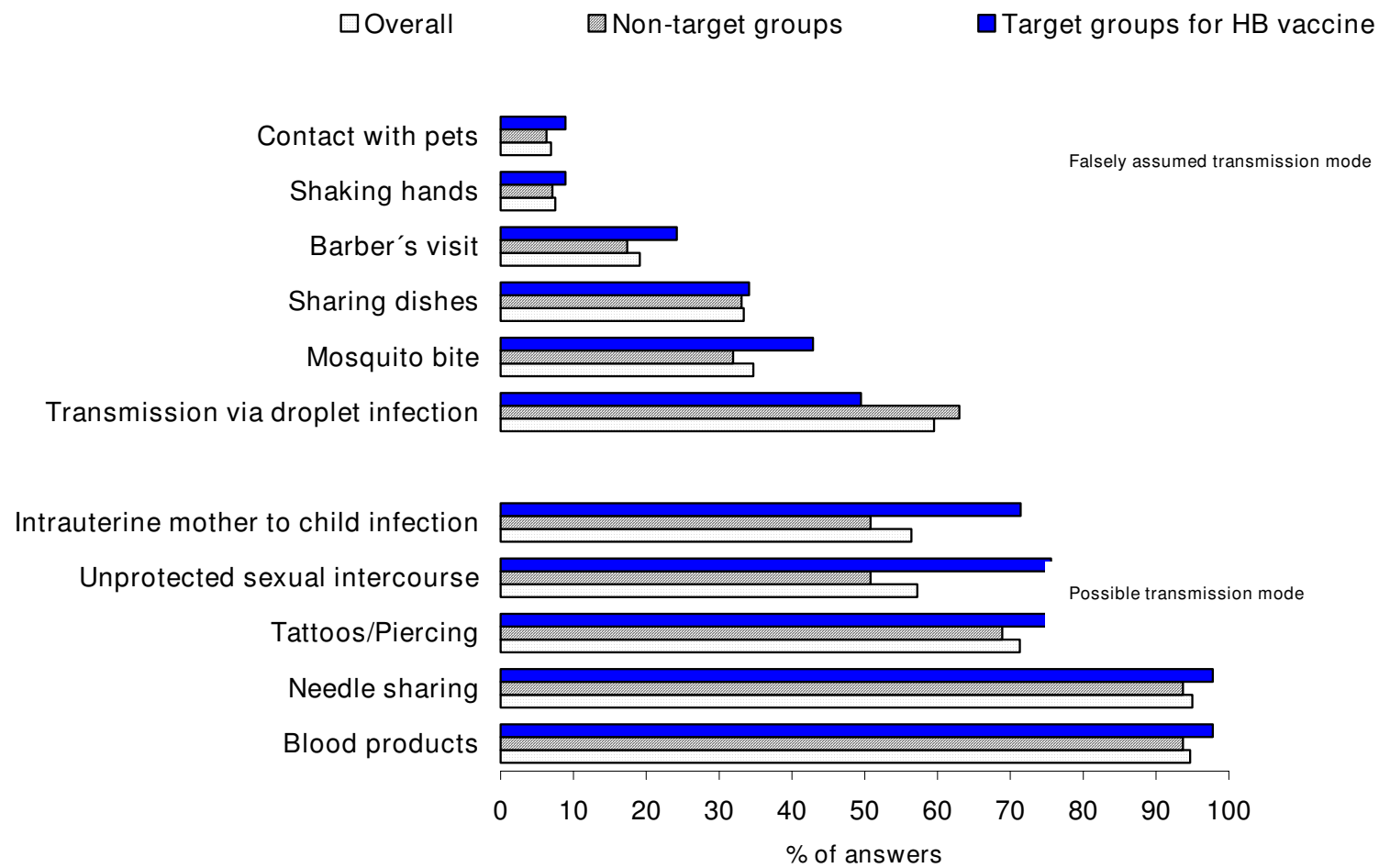

Figure 3

Knowledge on transmission modes for HB (all participants, target groups for HB vaccination, other participants). Standardised results (sex, age group and residency (East/West Germany) November 2004, $(n=4 / 2)$
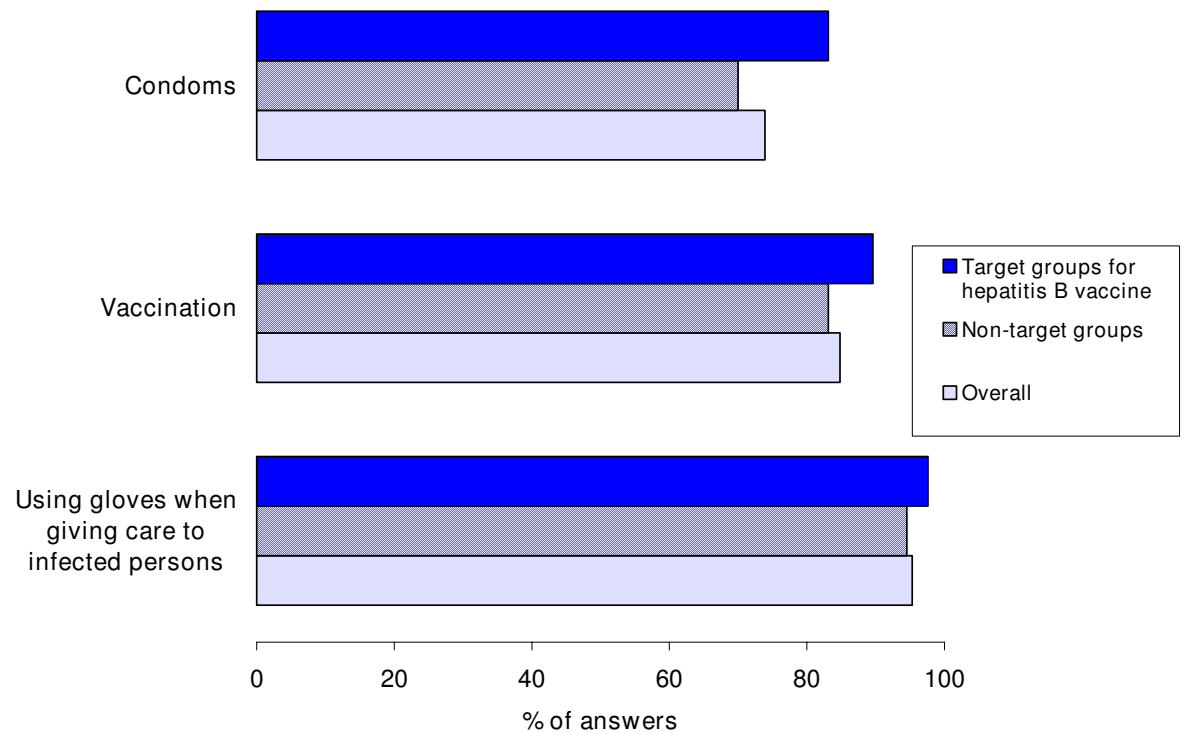

Figure 4

Knowledge about protective measures to avoid HB (overall sample, target groups for HB vaccination, other population groups). Standardised results (sex, age group and regional distribution), November $2004(n=4 I 2)$ 


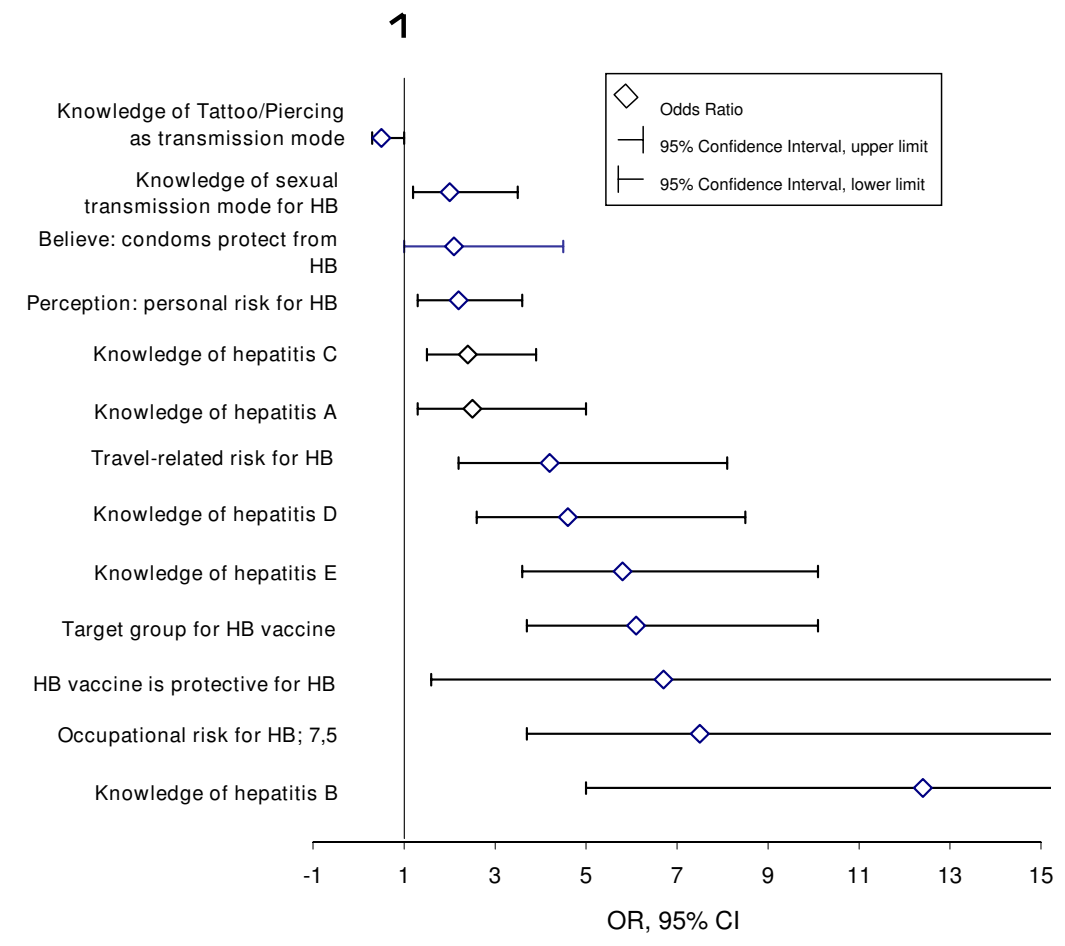

\section{Figure 5}

Point estimate (OR) and $\mathbf{9 5 \%}$ confidence intervals of factors associated with. positive vaccination status (bivariate analysis) Non-standardised results, Germany, November $2004(n=4 I 2)$

Since we classified a "vaccinated" person as someone reporting at least one vaccination against $\mathrm{HB}$ previously, one limitation of our study is the reliability on reported vaccination against $\mathrm{HB}$ without crosschecking their vaccination cards or GP records, which reportedly gives rise to some inaccuracy [13]. Particularly, there is the risk of misclassification as it might be possible that vaccination against hepatitis A was mistakenly assumed as vaccination against HB. Moreover, the exact number of vaccinations against $\mathrm{HB}$ might not have been recalled correctly or have been mixed up in single cases. However, for reasons of resource-effectiveness and practicability we had to rely on self-reported data. Also, the percentage of interviewees reporting to have had complete vaccination is quite high and many of them must have received the combined HA/ $H B$ vaccine; therefore, any misclassification due to mixing up $H A$ and $H B$ vaccine should be rare.
In spite of the fact that we chose a rather conservative estimate for "vaccination" (participants undecided about their vaccination status were treated as "not vaccinated") we believe that the observed vaccine coverage in the included target groups does not meet the expectations at all.

During our survey, we confined affiliation to target groups for HB vaccination to such groups with either an occupational, travel-related or illness-related risk for $\mathrm{HB}$ infection only. Other target groups, such as men who have sex with men (MSM) or i.v.-drug users were not taken into account during interviews, as these individual exposures are difficult to assess during a cross-sectional telephone survey. These subgroups deserve more attention, and further studies focussing on MSM or i.v.-drug users are needed in order to estimate vaccination coverage in these target groups, for which little data exist.

Table 4: Factors associated with positive vaccination status against HB. (Multivariate analysis) Germany, November $2004(\mathrm{n}=4 \mathrm{I} 2)$

\begin{tabular}{|c|c|c|c|}
\hline Factors associated with positive vaccination status & OR & $95 \% \mathrm{Cl}$ & $\mathrm{p}$-value \\
\hline Occupational risk for $\mathrm{HB}$ infection & 5.6 & $2.0-15.8$ & 0.001 \\
\hline Travel risk for HB infection & 5.4 & $2.0-14.7$ & 0.001 \\
\hline Age group (incremental) & 0.8 & $0.6-0.9$ & 0.037 \\
\hline
\end{tabular}


Table 5: Independent predictors of "good knowledge" on HB. (Multivariate analysis) Germany, November 2004, (n = 4I 2)

\begin{tabular}{|c|c|c|c|}
\hline Factors associated with good knowledge on HB & OR & $95 \% \mathrm{Cl}$ & p-value \\
\hline Occupational risk for HB infection & 11.5 & $2.5-52.8$ & 0.002 \\
\hline Positive attitude towards vaccinations in general & 5.4 & $1.2-19.9$ & 0.021 \\
\hline Increasing age group & 0.8 & $0.6-0.9$ & 0.008 \\
\hline Female gender & 0.5 & $0.3-0.9$ & 0.019 \\
\hline Interaction term [education level * sex] & 2.4 & $1.2-4.6$ & 0.016 \\
\hline
\end{tabular}

Occupational exposure for $\mathrm{HB}$ infection and travel-related exposure were associated factors with positive vaccination status in adults, indicating which conditions act as a fair reason to become vaccinated. However, since all health care workers should be vaccinated, the observed coverage in Germany is disappointingly low. In contrast, vaccination coverage in Health care workers in Switzerland, Belgium and Sweden were reported to range between $79 \%$ and $94 \%$ (at least one full dose vaccine received) [14-16]. However, concerning the study about health workers in Sweden the fact that health workers at risk only were included needs mentioning. This could have led to a generally higher estimate of vaccination coverage [15].

Chronically ill people were far less often vaccinated than travellers or occupationally exposed persons, which suggests that health care messages for these subgroups have not come across - be this founded in poor counselling or poor adherence. Also, a lack of knowledge among health staff might explain the low vaccination coverage of the chronically ill.

We confined the general term "good knowledge" on HB to a combined good knowledge about the major transmission routes, protective measures and types of HB. This rather specific definition for good knowledge could have lead to an underestimation of knowledge in the German population. However, this term was used for our model only but still particular subgroups of knowledge have nevertheless been presented within the descriptive analysis.

Our results on transmission risks for HB show that within the German population little knowledge exists about the fact that HB is transmitted sexually. This finding complies

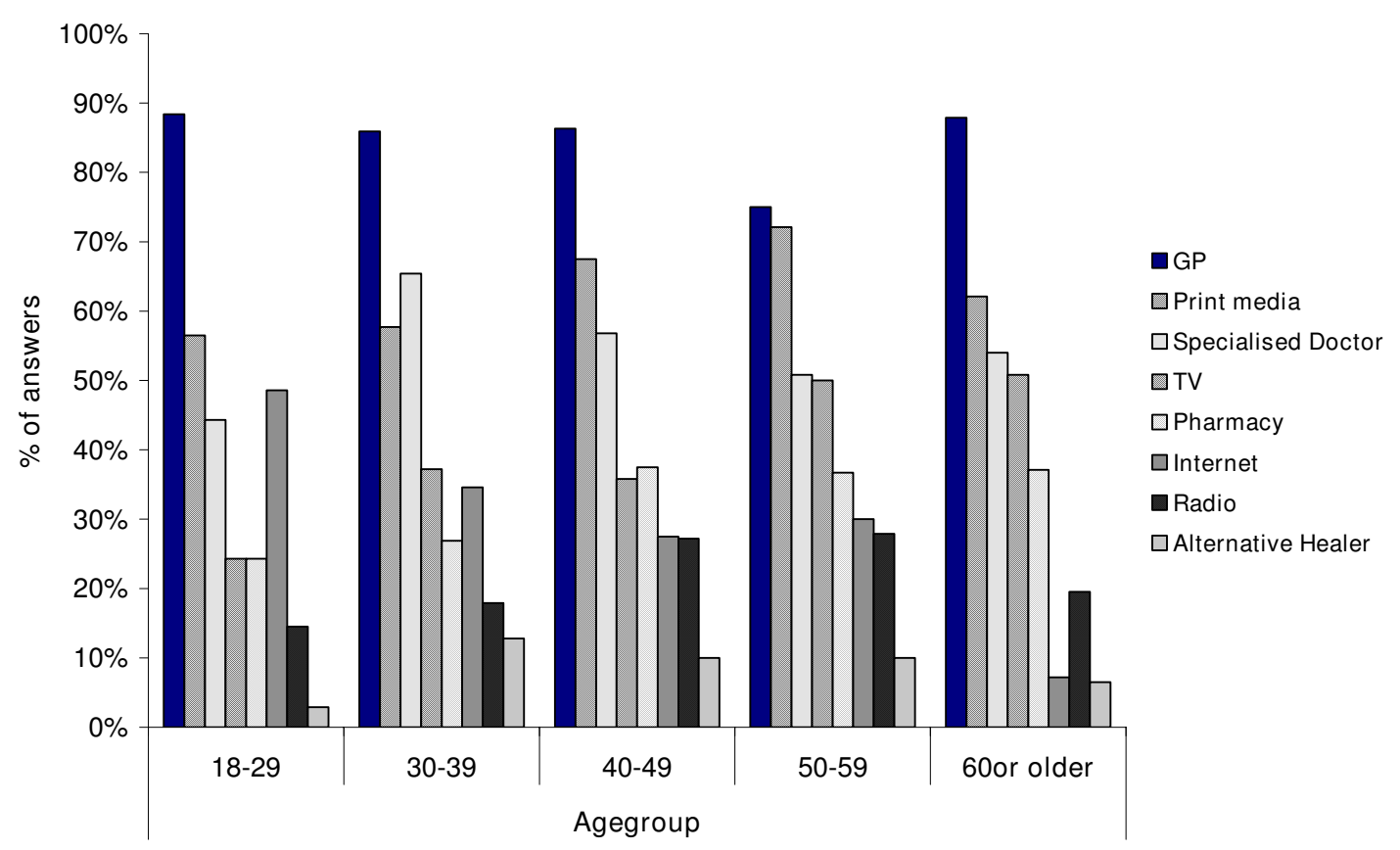

Figure 6

Sources of information on vaccine related issues by age group. Standardised results (sex, age group and regional distribution), Germany, November 2004 ( $n=412$ ) 
with the fact that condoms were less well known to protect from HB infection than hygiene measures and vaccination.

Age was negatively associated with "good knowledge" in our study which may reflect a better general health related knowledge in the young, or it may be due to employees such as health care workers with a better expertise. Furthermore, the fact that since $1995 \mathrm{HB}$ vaccination is recommended for all newborns and adolescents may have caused an increased awareness in those who are now young adults.

Corresponding to better knowledge among the young, age was inversely associated with vaccination status - the older participants were, the less likely they were to be vaccinated. This finding corresponds to a recent study on $\mathrm{HB}$ vaccination status among adult blood donors in Germany, where younger age was significantly associated with a higher $\mathrm{HB}$ vaccine coverage [17]. Also, this might be explained by the recent vaccine recommendation policy to vaccinate infants, children and adolescents against $\mathrm{HB}$ or may display a generally better acceptance of the HB vaccination [8].

Our findings are supported by preliminary results of another recent study on knowledge about hepatitis B in Germany which likewise revealed a clear association of social status and knowledge of $\mathrm{HB}$ as well as a negative association of age and knowledge of HB [18]. We identified a negative association of female gender and knowledge about $\mathrm{HB}$, although the association could be at least partly explained by an interaction between lesser school education and female sex. Also, a generally better knowledge of HB among MSM and iv drug users (more likely men) in our sample may have confounded our finding that men had a better knowledge of HB than women. In our sample population of 412 persons we expect for instance around $12 \mathrm{MSM}$ (5\% of 247 men in the sample population). Nevertheless, this finding is in contrary to the results of another German study where knowledge on transmission of disease and the use of preventive measures as immunisation was found better in females $(78 \%$ in females versus $69 \%$ in males, $\mathrm{p}=0.05$ ) [19]. Even after taking into account that the latter study involved students only and might therefore be biased by a generally higher education level it remains unclear why in our study knowledge was found better in men.

We observed a predominantly good appreciation of the protective effectiveness and safety of the $\mathrm{HB}$ vaccine and therefore assume that the general acceptance of $\mathrm{HB}$ vaccination within the German population is basically good; educational programmes on $\mathrm{HB}$ and vaccines are likely to lead to higher vaccination coverage in target groups for $\mathrm{HB}$ vaccine.

It has been shown that health-educational interventions regarding knowledge of $\mathrm{HB}$ risk factors and protective measures in students could measurably increase knowledge on $\mathrm{HB}$ and acceptance of vaccination [20]. In another study, educational measures about HB were particularly effective in influencing vaccination attitude in men who have sex with men when these programmes were able to increase the individual risk perception [21]. It can be hypothesized that this mechanism would work in other target groups, too.

In designing health-educational programmes one needs to bear in mind the low awareness of sexual transmission modes for $\mathrm{HB}$ in the general population, and that the protective effects of condom use, besides vaccination, should therefore be highlighted.

As poorer knowledge was found among higher age groups, information campaigns on HB should not only focus on the young, although they need to be tailored specifically for adolescents and young adults, in whom the $\mathrm{HB}$ incidence is still highest $[6,10]$.

$\mathrm{HB}$ vaccine uptake in those for whom $\mathrm{HB}$ vaccination is recommended but who tend to remain unvaccinated despite existing policies needs to be increased. This can be achieved by awareness campaigns and counselling.

As GPs and family doctors play a key role as a source for information, future educational measures concerning $\mathrm{HB}$ should be closely coordinated with the GP level of ambulatory care. It can be expected that counselling for vaccine issues could be performed far more efficiently by German GPs if they would be rewarded appropriately. Besides time restraints and poor payment, possible other reasons for insufficient information transfer at the GP level in Germany should be investigated more thoroughly.

\section{Conclusion}

Knowledge about HB and its risk factors in Germany was far below expectations and needs to be improved. Also, vaccination coverage in target groups was unsatisfactory. We conclude that educational measures could lead to a higher vaccine uptake in adult target groups.

\section{Competing interests}

The authors declare that they have no competing interests.

\section{Authors' contributions}

KS has prepared the study protocol, organised and supervised the telephone survey, data entry and data cleaning, conducted the data analysis and drafted the main manu- 
script. DR and VB have assisted in drafting the study protocol, co-supervised the telephone survey, assisted in the data analysis and contributed from the beginning in the internal draft reviewing. NB has co-supervised the telephone survey and assisted in data analysis and drafting the study protocol and main paper. Osamah Hamouda assisted in preparing the telephone survey and assisted in drafting the study protocol and later the main paper. All authors have approved the final manuscript.

\section{Acknowledgements}

The authors would like to thank all participants of the course on Applied Epidemiology of Infectious Diseases, held in November 2004 by the Robert Koch-Institute in Berlin for conducting the telephone interviews. Also, we thank the Health State office in Baden-Wuerttemberg especially Dr. Iris Zoellner. Finally, we would like to thank Dr. Sabine Häder and Dr. Sarika Desai.

\section{References}

I. Alter M: Epidemiology of viral hepatitis and HIV co-infection. $J$ Hepatol 2006, 44(I Suppl):6-9.

2. McMahon B]: Epidemiology and natural history of hepatitis B. Semin Liver Dis 2005, 25(Suppl I):3-8.

3. Fitzsimons D, Francois G, Alpers K, Radun D, Hallauer J, Jilg W, Gerlich W, Rombo L, Blystad H, Nokleby H, et al.: Prevention of viral hepatitis in the Nordic Countries and Germany. Scand J Infect Dis 2005, 37(8):549-560.

4. Jilg W, Hottentrager B, Weinberger K, Schlottmann K, Frick E, Holstege A, Scholmerich J, Palitzsch KD: Prevalence of markers of Hepatitis B in the adult German population. I Med Virol 200I, 63(2):96- 102.

5. Thierfelder W, Hellenbrand W, Meisel H, Schreier E, Dortschy R: Prevalence of markers for hepatitis A, B and C in the German population. Results of the German National Health Interview and Examination Survey 1998. Eur J Epidemiol 200I, I 7(5):429-435.

6. RKI: Infektionsepidemiologisches Jahrbuch für 2004. Berlin 2005.

7. Walter J, Radun D, Claus H, Hamouda O, Stark K: Hepatitis B and C risk factors in Germany-results of the national surveillance. Gesundheitswesen 2005, 67(7):44I-447.

8. RKI: Empfehlungen der Ständigen Impfkommission (STIKO) am Robert Koch-Institut, Stand: Juli 2005. Epidemiologisches Bulletin 2005:257-272.

9. RKI: Virushepatitis B, C und D: Situationsbericht 2005. Epidemiologisches Bulletin 2006:399-407.

10. RKI: Virushepatitis B, C und D: Situationsbericht 2004. Epidemiologisches Bulletin 2004:422-429.

II. Gabler S, Häder S: Telefonstichproben. Methodische Innovationen und Anwendungen in Deutschland Ist edition. Münster, New York, München: Waxmann Verlag; 2002.

12. Press release of the German Institute of Economic Research [http://www.diw.de/deutsch/pressemitteilungen/ 27284? pcode $=921$ \&language $=$ deutsch $]$

13. Wallaschofski $\mathrm{H}$, Drexler $\mathrm{H}$, Schmid K: What do medical students know about their vaccination status and how effective is their vaccine protection? Dtsch Med Wochenschr 2005, I30(23): 1429-1433.

14. BAG SEBfG: HIV-, HBV- und HCV-Expositionen im medizinischen Bereich in der Schweiz von 1997 bis 2000 . Bulletin des Bundesamtes für Gesundheit 2002, 40:692-696.

15. Dannetun E, Tegnell A, Torner A, Giesecke J: Coverage of hepatitis B vaccination in Swedish healthcare workers. The Journal of hospital infection 2006, 63(2):20I-204.

16. Vranckx R, Jacques P, De Schrijver A, Moens G: Hepatitis B vaccination coverage in Belgian health care workers. Infection 2004, 32(5):278-28।.

17. Ringwald JMI, Zimmermann R, Weisbach V, Rabe C, Strasser E, Seyboth S, Eckstein R: Hepatitis B vaccination status among healthy adults in Germany. Health Policy 2006:306-3I2.
18. Weitkunat R, Schlipköter U: Wissensstand der Bevölkerung über Hepatitis. Kompetenznetz Hepatitis/HepNet News 2005:4-6.

19. Klein SBA, Mossakowski K, Schipf S, Reintjes R: Health threats: Risk perception, knowledge and use of preventive measures among students in Hamburg. 50 Jahrestagung der Deutschen Gesellschaft für Medizinische Informatik, Biometrie und Epidemiologie (gmds) 12 Jahrestagung der Deutschen Arbeitsgemeinschaft für Epidemiologie (dae). Freiburg 2005.

20. Layer C, Gille G, Klapp C, Ravens-Sieberer U: [Prevention of hepatitis $B$ in juveniles. Effect of a medical health education lesson in school]. Med Klin 2004, 99( I 2):703-707.

21. De Wit JB, Vet R, Schutten M, van Steenbergen J: Social-cognitive determinants of vaccination behavior against hepatitis $B$ : an assessment among men who have sex with men. Prev Med 2005, 40(6):795-802.

\section{Pre-publication history}

The pre-publication history for this paper can be accessed here:

http://www.biomedcentral.com/1471-2458/8/132/pre pub

Publish with Bio Med Central and every scientist can read your work free of charge

"BioMed Central will be the most significant development for disseminating the results of biomedical research in our lifetime. " Sir Paul Nurse, Cancer Research UK

Your research papers will be:

- available free of charge to the entire biomedical community

- peer reviewed and published immediately upon acceptance

- cited in PubMed and archived on PubMed Central

- yours - you keep the copyright 